American Journal of Applied Sciences 6 (5): 834-842, 2009

ISSN 1546-9239

(C) 2009 Science Publications

\title{
Exact Simulating of Human Arteries using Lumped Model and Probing Constriction in Femoral and Carotid Arteries
}

\author{
Mohammad Reza Mirzaee, Omid Ghasemalizadeh and Bahar Firoozabadi \\ Department of Mechanical Engineering, \\ Sharif University of Technology Tehran, Tehran, Iran
}

\begin{abstract}
Problem statement: Modeling Human cardiovascular system was always an important issue from long past because by doing such modeling, investigation in cardiovascular system and its abnormities would be simpler. One of the most effective ways to do such modeling is using lumped method (Electrical analogy). Approach: Lumped method was used for simulating a complete model in this approach. A 36-vessel model was chosen to make equivalent circuit of lumped method from it. This complicated circuit includes equivalent segment for arteries, pulmonary, atrium, left and right ventricles. Furthermore, in this complex circuit some additional points were considered to improve this method. Some of the most important considerations to advance this modeling method was exact simulation of right and left ventricles pressures, division of ascending aorta, femoral carotid in respect into $27,10,31$ segments and adding peristaltic motions of vessels in descending arteries (thoracic to femoral) as a further pressure supplier respect to ventricles. Results: As a result of these considerations the pressure graphs from our complex circuit in descending arteries were so close to real graphs from biological texts which were earned by advanced instruments. Conclusion: citation of current modeling would be verified strongly. Consequently obstruction in femoral and right common carotid was probed with this complicated circuit and its results were shown in this study.
\end{abstract}

Key words: Electrical analogy (lumped method), cardiovascular systems, descending arteries, peristaltic motion of vessels walls, RLC circuit-obstruction

\section{INTRODUCTION}

Human cardiovascular system health and its abnormities are so important for clinical researches. Because by knowing the influences of diseases on cardiovascular system properties such as pressure graph the prevention from serious health problems would be possible. To analyze cardiovascular system and effects of diseases on it different ways are usable such as lumped model, one or multi-dimensional modeling and experimental methods. In this context lumped method were used with the goal of providing better understanding and simulation of the blood flow in the human cardiovascular system which will lead to accurate answers as a result of exact modeling. The first computer models describing the arterial system such as ascending aorta and carotids were introduced a multibranched model of the arterial tree in a usable form for digital computers. By this method different physiological conditions became considerable. Later more detailed models were applied to reach more accurate results ${ }^{[1]}$. To analyze the human cardiovascular system mathematically, more simplified model should be considered to decrease the difficulty of investigating. A pulsatile-flow model of the left and right ventricles (as suppliers) and 2-segment aorta were constructed and the changes in flow behavior investigated. Later lumped (electrical analogy) model was developed to analyze cardiovascular systems easily with suitable accuracy. An electrical model which focused on the vessel properties was made by Young and his team ${ }^{[2]}$. Onedimensional axisymmetric Navier-Stokes equations for time dependent blood flow in a rigid vessel had been used to derive lumped models relating flow and pressure $^{[3]}$. The effect of drugs on the blood properties and its circulation was studied later in a 19compartment model ${ }^{[4]}$. A complicated non-linear computer model for pressure changes and flow propagation in the human arterial system was drived ${ }^{[5]}$. The model had 55 arterial compartments and was based on one-dimensional flow equations to simulate effects of hydrodynamic parameters on blood flow. A Computational model was developed to provide boundary conditions for simulations of the effects of endoleak on the AAA wall stress ${ }^{[6]}$. later a simple model of lumped method was presented for human body ${ }^{[7]}$.

Corresponding Author: Omid Ghasemalizadeh, Department of Mechanical Engineering, Sharif University of Technology Tehran, Tehran, Iran 
This study describes modeling of the whole human cardiovascular system using an extensive equivalent electronic circuit. In this method we have taken a quite different way to model the system and we had intention to develop our electrical models by addition more segments to main arteries which more accurate results would be one of its effects. The model consists of about 150 RLC segments representing the arterial and cardiac systems. Compared to previous studies, both arterial and cardiac systems of this model are more detailed, especially femoral, ascending aorta and upper arteries such as carotid, brachial and Brachiocephalic. So more exact results for ascending aorta and other named vessels properties could be reachable. Logically the method of increasing the number of segments for each artery will increase the accuracy of answers greatly. Also investigating cardiovascular system faults and effects of diseases would cause precise results. In addition using more compartments to model each artery creates this possibility to simulate variable radius of each artery quite exactly. Therefore because of considering variable radius, abnormities such as obstructions in different arteries can be probed quite real.

So as the main purpose of this research obstruction in femoral and right common carotid arteries is considered and its influences on pressure of main arteries are shown.

Here is a 36-vessel body tree which is utilized in this research to model human cardiovascular system vessels. The number of each part point to its equivalent circuit. Also information about these vessels is shown in Fig. 1 and Table 1.

Modeling principles and considerations: To model human cardiovascular system we chose different equivalent electrical elements to express different mechanical properties of vessels, blood and heart.

In our modeling process atriums, ventricles, every blood vessel, set of all capillaries, arterioles and veins have been presented by some compartments consisting of a resistor, an inducer and a capacitor.

The number of compartments would be chosen by the purpose and the required accuracy .So more compartments would be used for main arteries.

Voltage, current, charge, resistance and capacitance, inductor in the electronic circuit are respectively equivalent to blood pressure, blood flow, volume, resistance, compliance and flow inertia in the cardiovascular system. Ground potential (reference for voltage measurements) is assumed to be zero as usual. The relation between mechanical properties of

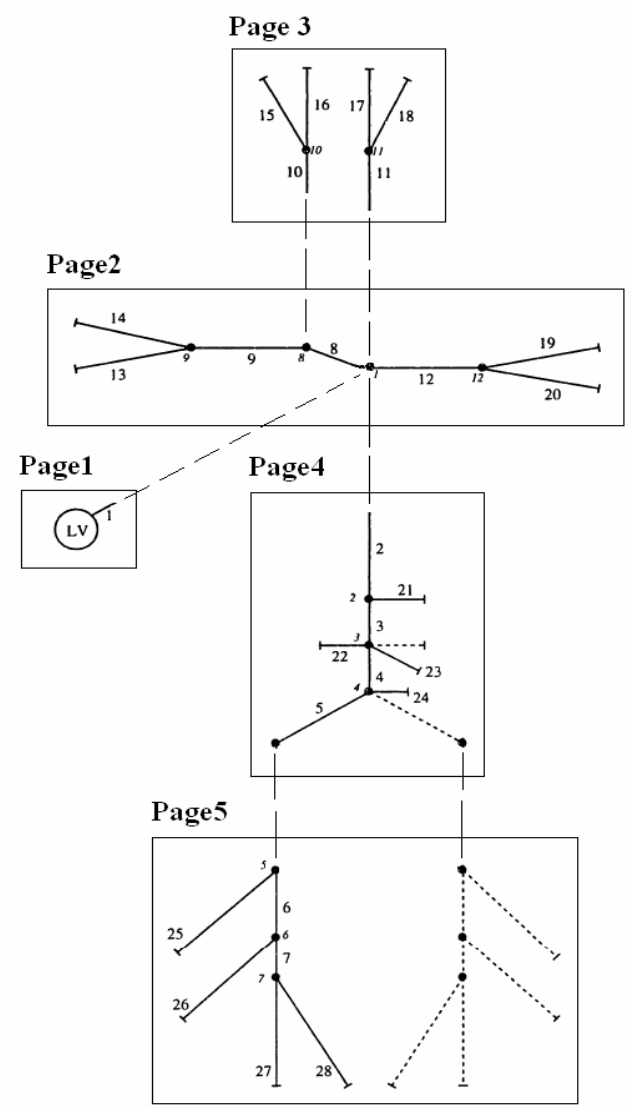

Fig. 1: 36-Vessels body tree

cardiovascular system and their equivalent electrical elements are as follow:

$0.01 \mathrm{~mL} / \mathrm{Pa} \quad=1 \mu \mathrm{F}$ (compliance-capacitance)

$1 \mathrm{~Pa} \cdot \mathrm{sec}^{-2} \mathrm{~mL}^{-1}=1 \mu \mathrm{H}$ (inertia-inductor)

$1 \mathrm{~Pa} . \sec \mathrm{mL}^{-1}=1 \mathrm{k} \Omega$ (resistance-resistance)

$1 \mathrm{~mm} \mathrm{Hg} \quad=1$ volt (pressure-voltage)

$133416 \mathrm{~mL}=1 \mathrm{~A}(\text { volume-charge })^{7}$

Following formulas is taken to introduce needed electrical elements for simulation ${ }^{[8]}$.

Blood vessel Resistance (R), depending on blood viscosity and vessel diameter, is simulated by resistors:

$$
\mathrm{R}=\frac{81 \pi \mu}{\mathrm{A}^{2}}
$$

where $\mu$ is blood viscosity, 1 and $\mathrm{A}$ are in respect length and cross section area of each artery segment.

This simulation has considered because blood viscosity will cause resistance against Blood flow crossing. 
Am. J. Applied Sci., 6 (5): 834-842, 2009

Table 1 - Calculated Values for Elements of Circuit from artery parameters

\begin{tabular}{|c|c|c|c|c|c|c|c|c|c|}
\hline No. & Vessel Name & $\mathrm{A}\left(\mathrm{cm}^{\wedge} 2\right)$ & $1(\mathrm{~cm})$ & $\mathrm{h}(\mathrm{cm})$ & $\mathrm{n}$ & E (Mpa) & $\mathrm{R}(\mathrm{K} \Omega)$ & $\mathrm{C}(\mu \mathrm{F})$ & $\mathrm{L}(\mu \mathrm{H})$ \\
\hline 1 & Ascending Aorta & 6.60507 & 0.204 & 0.163 & 27 & 0.4 & 0.000411 & 0.044883 & 0.007286 \\
\hline 2 & Thoracic Aorta & 3.59701 & 18.5 & 0.124 & 1 & 0.4 & 0.125776 & 2.15337 & 1.215072 \\
\hline 3 & Abdominal Aorta & 2.378 & 4.3 & 0.11 & 1 & 0.4 & 0.066889 & 0.303284 & 0.427197 \\
\hline 4 & Abdominal Aorta & 1.021 & 9.6 & 0.08 & 1 & 0.4 & 0.810079 & 0.261925 & 2.221352 \\
\hline 5 & Common iliac & 0.849 & 19.2 & 0.076 & 1 & 0.4 & 2.343114 & 0.418125 & 5.342756 \\
\hline 6 & Femoral Artery & 0.181 & 5.4 & 0.048 & 8 & 0.8 & 14.4992 & 0.009164 & 7.048343 \\
\hline 7 & Anterior Tibial Artery & 0.053 & 1.5 & 0.035 & 1 & 1.6 & 46.97287 & 0.000277 & 6.686321 \\
\hline 8 & Brachiocephalic & 1.20798 & 2.4 & 0.085 & 1 & 0.4 & 0.144677 & 0.079312 & 0.469379 \\
\hline 9 & R Brachial & 0.50298 & 5.125 & 0.064 & 8 & 0.4 & 1.78196 & 0.060436 & 2.40721 \\
\hline 10 & R Common Carotid & 0.50298 & 2.8 & 0.064 & 6 & 0.4 & 0.973559 & 0.033019 & 1.315159 \\
\hline 11 & L Common Carotid & 0.50298 & 2.2 & 0.064 & 5 & 0.4 & 0.764939 & 0.025943 & 1.033339 \\
\hline 12 & L Brachial & 0.55399 & 44.4 & 0.066 & 1 & 0.4 & 12.72576 & 0.586882 & 18.9344 \\
\hline 13 & R Radial & 0.08 & 4.64 & 0.043 & 5 & 0.8 & 63.76945 & 0.002583 & 13.70198 \\
\hline 14 & R Ulnar & 0.13901 & 4.58 & 0.047 & 5 & 0.8 & 20.8499 & 0.005343 & 7.783999 \\
\hline 15 & R External Carotid & 0.196 & 2.26 & 0.049 & 5 & 0.8 & 5.174922 & 0.004234 & 2.724107 \\
\hline 16 & R Internal Carotid & 0.283 & 3.44 & 0.054 & 5 & 0.8 & 3.778271 & 0.010145 & 2.871731 \\
\hline 17 & L Internal Carotid & 0.283 & 3.44 & 0.054 & 5 & 0.8 & 3.778271 & 0.010145 & 2.871731 \\
\hline 18 & L External Carotid & 0.196 & 2.26 & 0.049 & 5 & 0.8 & 5.174922 & 0.004234 & 2.724107 \\
\hline 19 & L Radial & 0.13901 & 4.64 & 0.047 & 5 & 0.8 & 21.12304 & 0.005413 & 7.885973 \\
\hline 20 & L Ulnar & 0.08 & 4.58 & 0.043 & 5 & 0.8 & 62.94484 & 0.00255 & 13.5248 \\
\hline 21 & Coeliac & 0.478 & 1 & 0.064 & 1 & 0.4 & 0.384992 & 0.010925 & 0.494247 \\
\hline 22 & Renal & 0.212 & 2.7 & 0.049 & 1 & 0.4 & 5.284447 & 0.01138 & 3.008844 \\
\hline 23 & Sup Mesenteric & 0.581 & 5.4 & 0.066 & 1 & 0.4 & 1.407178 & 0.07666 & 2.195783 \\
\hline 24 & Inf Mesenteric & 0.08 & 4.5 & 0.043 & 1 & 0.4 & 61.85005 & 0.00501 & 13.28906 \\
\hline 25 & Profundis & 0.166 & 12.1 & 0.047 & 1 & 1.6 & 38.62573 & 0.00921 & 17.22063 \\
\hline 26 & Post Tibial & 0.102 & 30.6 & 0.043 & 1 & 1.6 & 258.7192 & 0.012262 & 70.875 \\
\hline 27 & Ant Tibial & 0.031 & 29.5 & 0.03 & 1 & 1.6 & 2700.264 & 0.002839 & 224.8185 \\
\hline 28 & Proneal & 0.053 & 31.3 & 0.035 & 1 & 1.6 & 980.1671 & 0.005771 & 139.5212 \\
\hline
\end{tabular}

The blood inertia (L) is simulated by inductors:

$$
\mathrm{L}=\frac{91 \rho}{4 \mathrm{~A}}
$$

where $\rho$ is blood density.

Reason of this consideration is variability of flow acceleration in pulsatile blood flow, so an inductor can model inertia of blood flow very clearly.

The vessel Compliance (C) is considered using capacitors:

$$
\mathrm{C}=\frac{31 \pi \mathrm{r}^{3}}{2 \mathrm{Eh}}
$$

where $\mathrm{r}, \mathrm{E}, \mathrm{h}$ are in respect artery radius, Elasticity module and thickness of arteries.

For the reason of this simulation, it should be noted that by passing blood thorough vessels, the vessels would be expanded or contracted, so they can keep blood or release it and this is exactly like what a capacitor does. By these statements each vessel is modeled by some compartments, which includes one resistance, one capacitor and one inductor. Quantities of Compartment's elements are easily achievable by using Eq. 1-3. Computed values of circuit Elements are shown in Table 1.

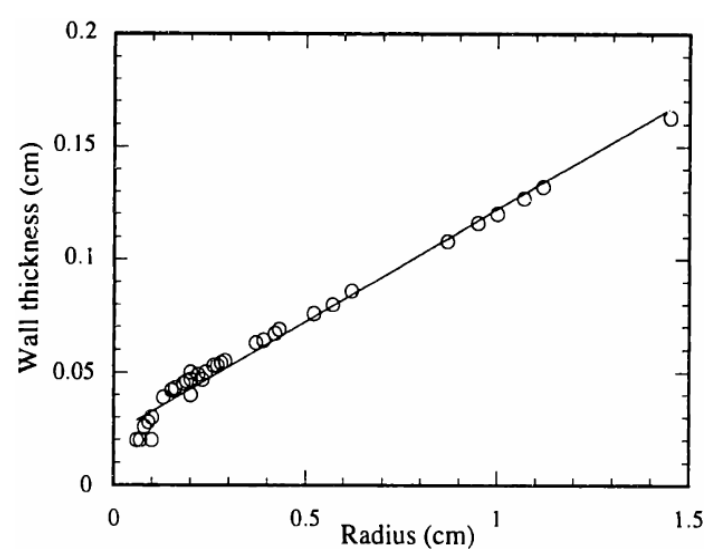

Fig. 2: Wall Thickness versus Radius

Where $\rho$ is $1050\left(\mathrm{~kg} \mathrm{~m}^{-3}\right), \mu$ is $0.0035\left(\mathrm{~kg} \mathrm{~m} \mathrm{sec}^{-1}\right)$ and $\mathrm{n}$ is number of each artery segments. We can obviously understand the density of blood is so near to water density, So their properties would be very similar.

Also, artery wall thickness (h) is obtained from Fig. 2. (Physiological text ${ }^{[9]}$ )

Atriums are simulated as part of the venous system without any contraction. Atriums and ventricles can be modeled like vessels as a RLC segment. These two important parts of heart have two courses of action, one is resting position (diastole) which muscles would take 


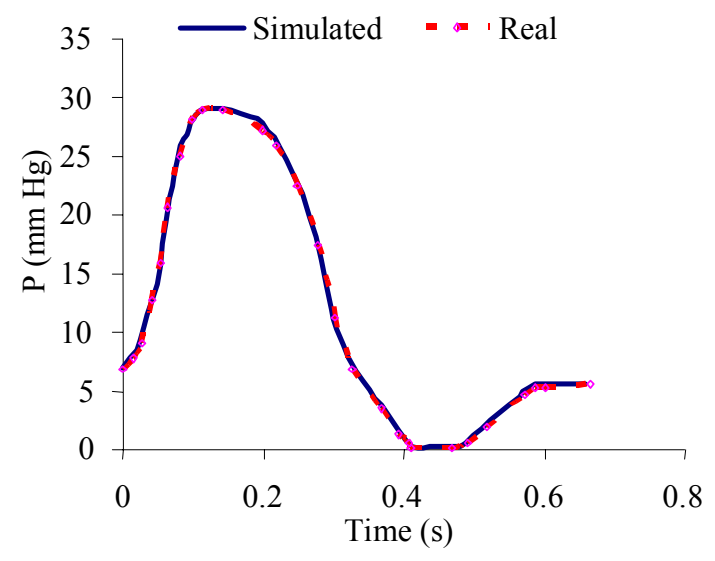

Fig. 3: Simulated (solid line) and real (doted line) pressure for right ventricle

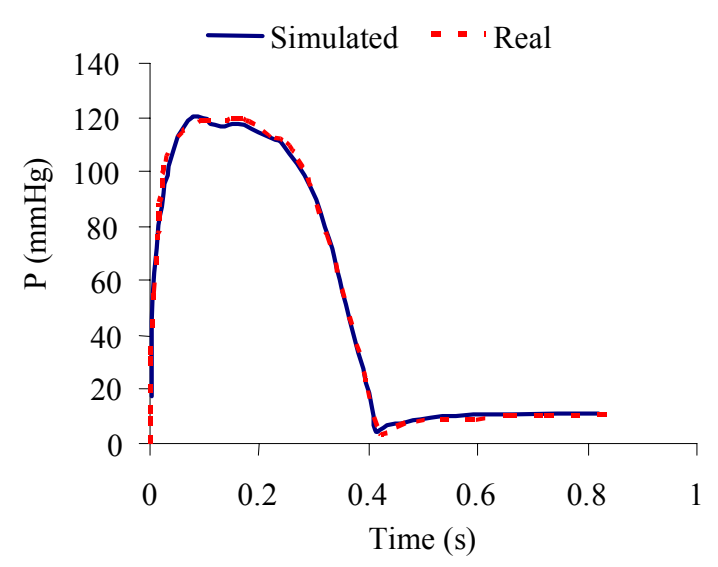

Fig. 4: Simulated (solid line) and real (doted line) pressure for left ventricle

their maximum volume and the other is acting position (systole) which muscles reach to their minimum size.

Also we should note that heart with its moving muscles is the power supplier of blood circulation in whole body vessels.

To have a very exact modeling of blood and vessels behaviors, exact modeling of power suppliers is an important factor. So in our simulation left and right ventricles are modeled quite exactly the same as biological graph sources.

Simulated and real graphs of right and left ventricles are shown in turn in Fig. 3 and 4 and they would be more explained in circuit description.

Different heart shutters are modeled by using appropriate diodes, because shutters like diodes cross the flow in one direction. Considering this fact is important because in some parts of cardiovascular system, inverse current movement will cause to great danger to health. So to reach a good model, choosing appropriate diodes would be counted as an inseparable part.

Also, bifurcations are important cases to have accurate modeling. For simulating these parts of cardiovascular system a special method has been used $^{[8]}$. In brief, it can be said that in bifurcations properties of jointed vessels will combine together in a complicated way to show the blood current division effects.

Peristaltic motion of vessels: When several simultaneous measurements are done at different points all along the aorta, it appears that the pressure wave changes shape as it travels down the aorta. Whereas, the systolic blood pressure actually increases with distance from the heart. Thus the amplitude of the pressure oscillation between systole and diastole, which is pulse pressure, nearly doubles. Thereafter, both PP (Pulse Pressure) and MAP (Mean Aorta Pressure) decrease rapidly ${ }^{[10]}$.

Logically, wastes because of frictions and bifurcations should be caused decreasement of systolic pressure of descending arteries but as said above from biological texts, it is obvious from the Fig. $5^{[10]}$. That by moving forward through these arteries the maximum point of pressure graph would be increased. The reason of this phenomenon is that these arteries have an additional pressure which is the result of waveform movement of vessels walls. These walls have a peristaltic motion which it would help the easier movement of blood in descending vessels.

To have more exact results from our circuit, in addition to precise simulation of left and right ventricles we used appropriate pressure suppliers to consider these peristaltic motions and their effects. To model these pressure suppliers in our electronic circuit for each artery and respect to its motion, appropriate voltage sources were chosen.

Obstruction modeling: In this section obstructions in important places would be modeled. In this research constriction in femoral and right common carotid would be had in mind. Real shape of obstruction in these two arteries is shown in Fig. $6^{[11]}$.

Also, simulated 3D model of obstruction for these arteries is shown in Fig. 7.

To have an exact obstruction model, we should note that this phenomenon doesn't occur in one point very sharply but it happens smoothly, it means the radius will decrease slowly and in one point it reaches to its minimum quantity, after that it begins to increase again to its first quantity (vessel radius without 


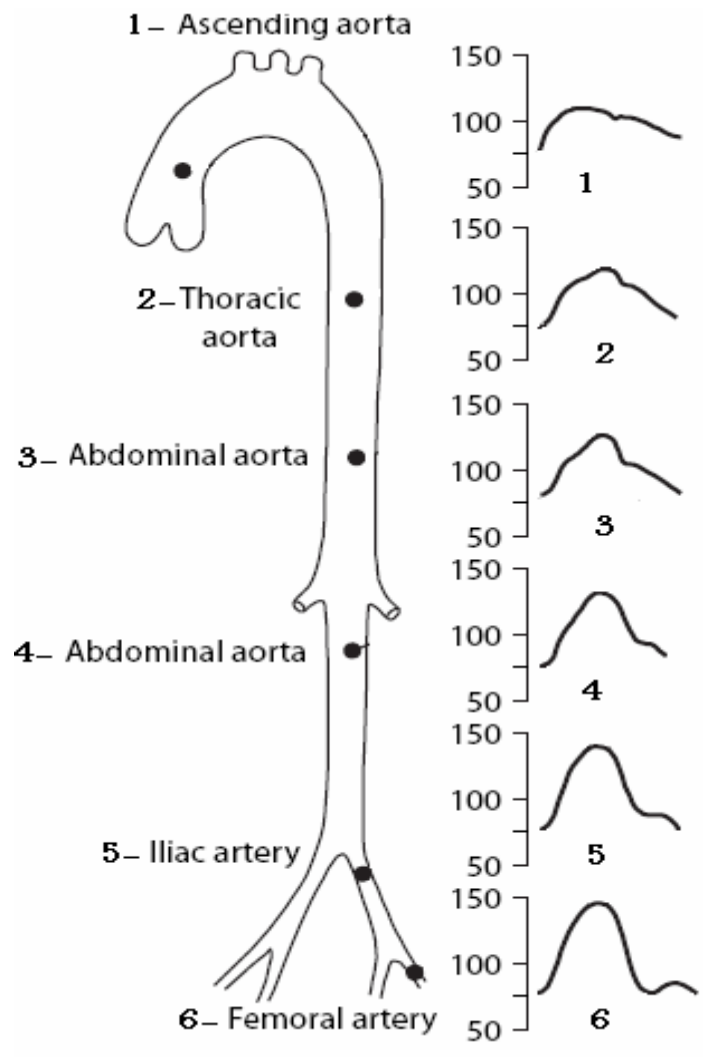

Fig. 5: The pressure changes thorough descending arteries caused by peristaltic motion

obstruction). So for each artery with respect to its length and its importance, numbers of segments would be chosen. These segments will show the changes of radius in the region of obstruction.

As said in physiological text ${ }^{[9]}$, femoral is 43.2 centimeters and in our circuit it has been modeled with 8 compartments. By above considerations, length of each compartment becomes 5.4 millimeters. So to model obstruction region we considered 16.2 centimeters length of femoral that includes 3 compartments with numbers 1-3. These compartments are significant in femoral circuit. The percent of constriction would be $70 \%$.

From the same physiological text ${ }^{[9]}$ it is obvious that right common carotid length is 16.8 centimeters and in equivalent circuit it has been modeled with 6 compartments which each segments length become 2.8 centimeters. To model obstruction for this artery, 1 segment would be chosen with $60 \%$ constriction which it is significant with number 4 in equivalent circuit. One segment is chosen because obstruction would not happen with much decreasement in radius along brachial vessel. It is obvious from the Fig. 6 .

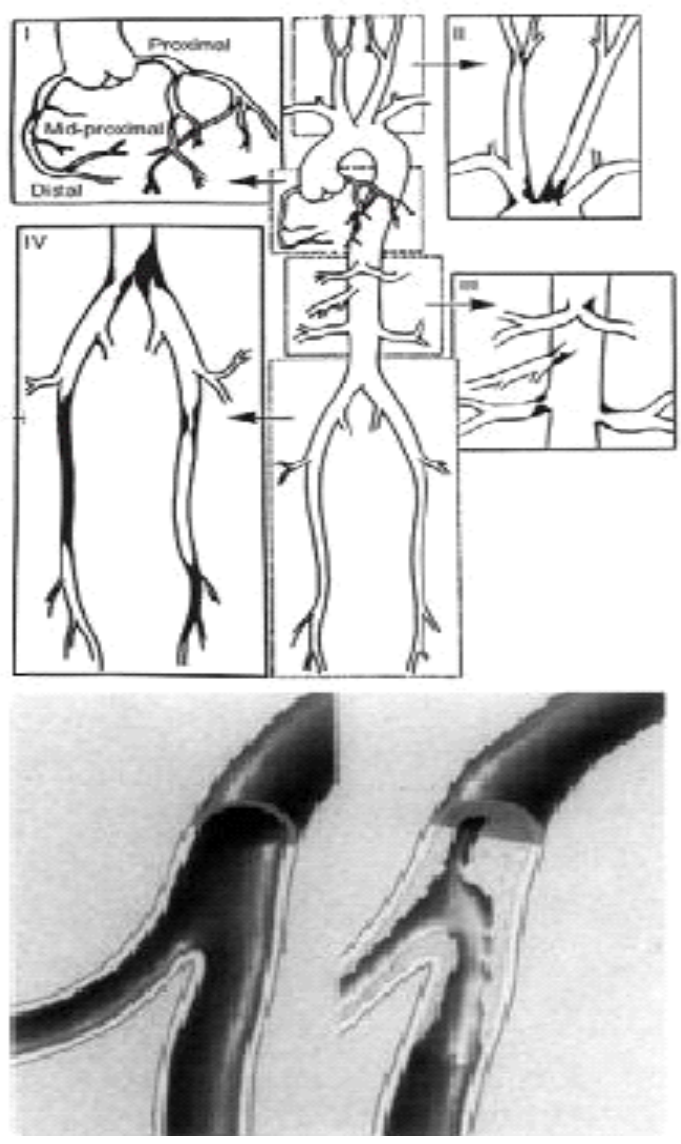

Fig. 6: Real shape of obstruction in femoral and right common carotid from texts

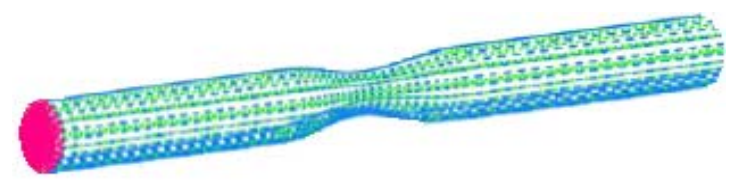

Fig. 7: 3D shape of obstruction in femoral and right common carotid

To calculate obstruction elements values, in according to Eq. 3, capacitor quantity is related to wall thickness and elasticity modulus. Values of these parameters would vary because of obstruction. Wall thickness (h) increases with respect to percent of obstruction and also, Elasticity modulus (E) variation would be computable with considering Eq. 4 .

$$
\mathrm{a}=\sqrt{\frac{E h}{2 \mathrm{r} \rho}}
$$

where (a) is wave propagation velocity in the vessel. 
Am. J. Applied Sci., 6 (5): 834-842, 2009

Table 2: Calculated values for vessel properties in obstruction

\begin{tabular}{lllll}
\hline Obstruction percentage & & $\mathrm{r}_{2}$ & $\mathrm{E}_{2}$ & $\mathrm{~h}_{2}$ \\
\hline Femoral & 70 & 0.07201 & 0.05333 & 0.2160 \\
& 50 & 0.12001 & 0.11428 & 0.1680 \\
Right Carotid & 60 & 0.16005 & 0.03368 & 0.3041 \\
\hline
\end{tabular}

Table 3: Calculated values for obstruction elements

\begin{tabular}{lllll}
\hline Obstruction percentage & & $\mathrm{R}(\Omega)$ & $\mathrm{L}(\mu \mathrm{H})$ & $\mathrm{C}(\mu \mathrm{F})$ \\
\hline Femoral & 70 & 1790039 & 0.0004 & 78.315 \\
& 50 & 231989 & 0.0011 & 28.193 \\
Right Carotid & 60 & 38029.6 & 0.0026 & 8.2197
\end{tabular}

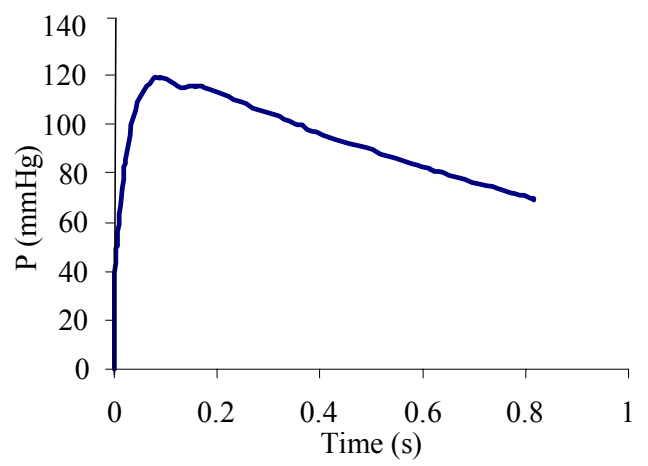

Fig. 8: Calculated pressure for ascending aorta

Note that (a) would remain constant because there is no change in vessel material. The computed values for $(\mathrm{r}),(\mathrm{h})$ and $(\mathrm{E})$ are given in Table 2.

By referring to Table 2, obstruction elements such as $\mathrm{R}, \mathrm{L}$ and $\mathrm{C}$ would be as follow in Table 3 .

Also equivalent Figures and circuits for obstruction in both named arteries are visible in Fig. 7 and 8.

Circuit description: Elements quantities of human cardiovascular system equivalent circuit which have been earned from modeling principles. This circuit includes five main parts that each one is a complicated circuit for one part of body.

- Heart and ascending aorta circuit

- upper body circuit

- carotid arteries circuit

- downer body circuit

- femoral arteries circuit

These additional segments for different parts of body are done because in each part of circuit increasing the number of compartments would close our answers to real values and it is because that this work will increase the number of capacitors. Consequently, their values would decrease and this will lead to lesser leakage of current.

\section{Heart and ascending aorta circuit:}

- Ascending aorta and heart (ventricles, atriums, pulmonary and shutters)

- Ascending aorta would be subdivided into 27 segments which elements quantities are shown in Table 1.

The right atrium and ventricle are modeled by two capacitors $216.45 \mu \mathrm{F}$ and $150 \mu \mathrm{F}$. Also the left atrium and ventricle are represented by two capacitors $101 \mu \mathrm{F}$ and $25 \mu \mathrm{F}^{[8]}$.

As said before we should use appropriate diodes to model shutters. Diodes used for tricuspid, pulmonary, mitral and aortic shutters are in respect 120NQ045, QSCH5545/- 55C, SD41 and SD41.

Also an exact model of ventricles pressure has been used as the supply of power. The simulated and exact pressure graphs have been compared in Fig. 3 for right ventricle and Fig. 4 for left ventricle.

From the figures it is obvious that left and right ventricles pressure will change, in turn between 120-11 volt $(\mathrm{mmHg})$ and $29-7$ volt $(\mathrm{mmHg})$.

\section{Arteries circuit except ascending aorta:}

- $\quad$ upper part of body (hands and carotids)

- carotid arteries circuit

- downer part of body (thoracic aorta and feet)

- femoral arteries circuit

As said before voltage suppliers would be considered to model motion of vessels muscles. This should just be added to important descending arteries from aorta such as thoracic, abdominal aorta, iliac and femoral arteries.

In human body Cardiac current output and aortic pressure should be in turn $100\left(\mathrm{~mL} \mathrm{sec}^{-1}\right)$ and 120-68 $(\mathrm{mmHg})^{[12]}$. These were exactly calculated in our circuit, so our modeling correctness is verifying undoubtedly.

For obstruction it should be noted, because each segment in obstruction region has its own radius, its elements quantities would be different and we should use formulas 1-3 separately to compute their amounts.

Additional explanation: It should be noted that capillaries are so small but have an important role in cardiovascular system which without them the circulation of blood would not be completed. In our circuit marker VCC3 plays role of capillaries which connect arteries to veins. If these parts of circuit don't 
Am. J. Applied Sci., 6 (5): 834-842, 2009

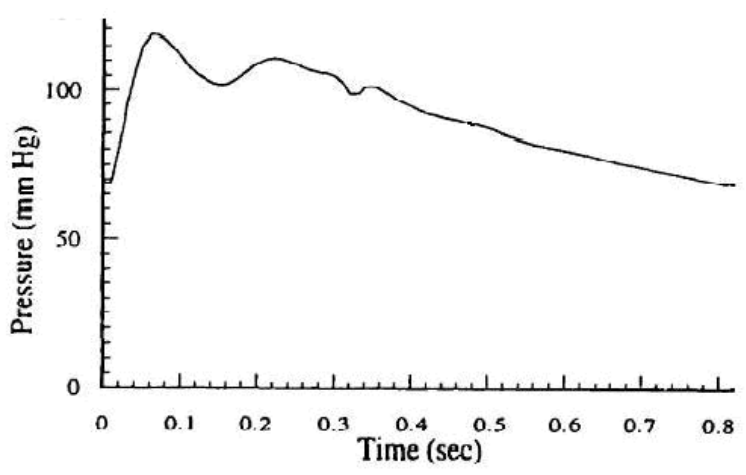

Fig. 9: Real pressure for ascending aorta

put correctly in their places, the modeling and its equivalent circuit wouldn't accompany real and exact answers.

It should be added that $\operatorname{VCC}(\mathrm{x}) \mathrm{s}$, allude to co potential points.

It should be noted that by using more than 80 segments to model whole arteries there is no leakage of current in the system and arteries pressure graph are quite acceptable comparing to the input pressure. It means blood flow after exiting from left ventricle and crossing from arteries and without decreasing of its quantity, would enter veins from capillaries. By power of right ventricle it will pump to pulmonary and will pour to left ventricle again so the cycle would be completed.

Also model is capable of showing blood and vessels properties in different points. For example the pressure (voltage) and volume (charge) graphs can be obtained from the different points of the circuit easily. But in this circuit we would quietly focus on pressure graphs that are productive in clinical researches.

Verification of circuit: In human body Cardiac current output and aortic pressure should be in turn $100 \mathrm{~mL}$ $\mathrm{sec}^{-1}$ and 120-68 (mmHg) ${ }^{[12]}$. The calculated current from our circuit is exactly the same as sources.

Also as we can see, the calculated pressure changes of ascending aorta artery with 10 compartments are shown in Fig. 8. This graph shows that aorta pressure varies between 120-68 mmHg (volt) (systole-diastole) and the results are in exact agreement with physiological article ${ }^{[9]}$ (Fig. 9). Even, two peaks in pressure graph of ascending aorta are earned the same as real measurements.

Because of these agreements between real and calculated graphs for ascending aorta, right and left ventricles the citation of our circuit and modeling would be verified.

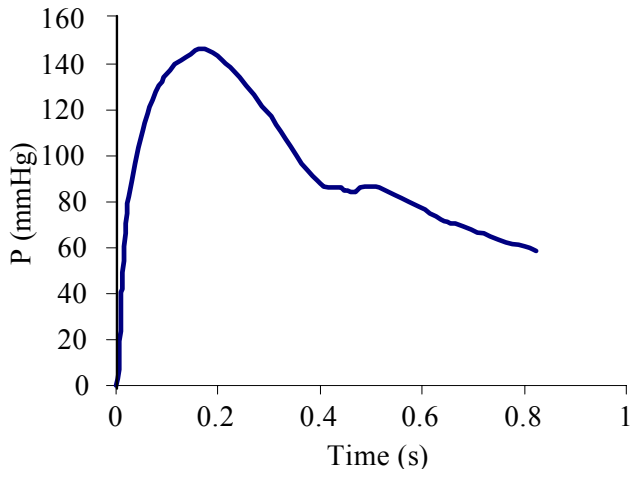

Fig. 10: Calculated pressure for femoral artery

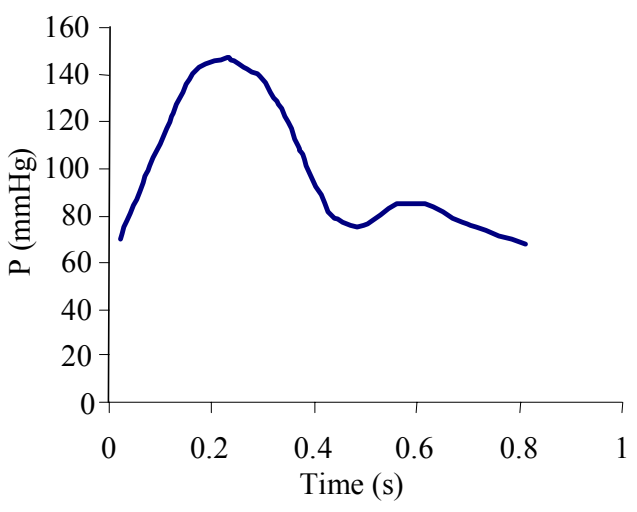

Fig. 11: Real pressure for femoral artery

In the next step confirmation of femoral pressure graph (Fig. 10) would be done and also pressure graph of carotid (Fig. 11) is shown after it.

The simulated and real pressure graphs of femoral are shown as follow.

At the end calculated pressure for carotid artery is shown below.

\section{RESULTS AND DISCUSSION}

According to above text, we can use verification part to make obstruction results quite citable.

For obstruction in femoral with 8 compartments, results are shown as below. A Femoral pressure graph in obstruction is shown in Fig. 12.

These Figures are measured before and after location of obstruction so increasement in pressure should be happen upstream of obstruction and it is obvious in our results.

Also, Pressure difference between normal and abnormal mode of femoral for $70 \%$ constriction is shown in Fig. 13. 
Am. J. Applied Sci., 6 (5): 834-842, 2009

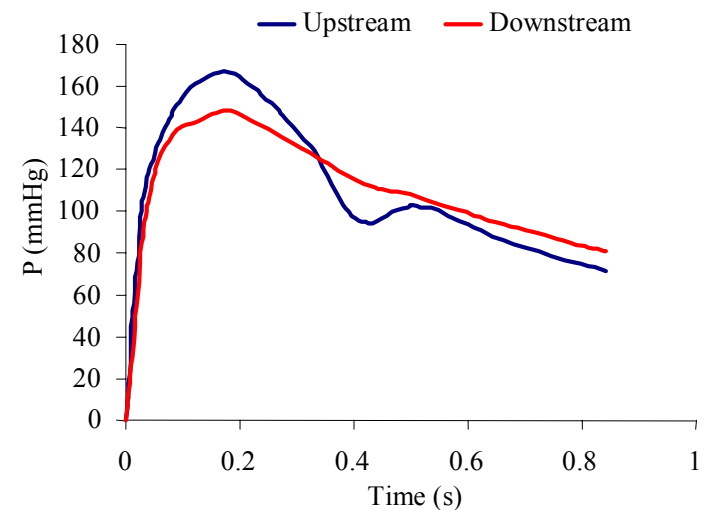

Fig. 12: Calculated pressure for abnormal mode of femoral

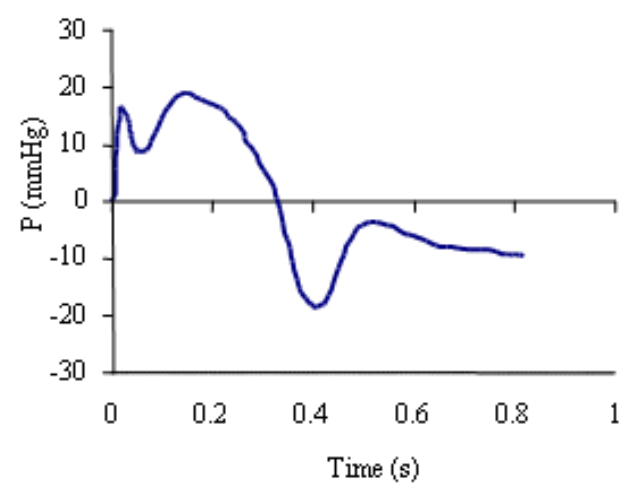

Fig. 13: Pressure difference between upstream and downstream of femoral

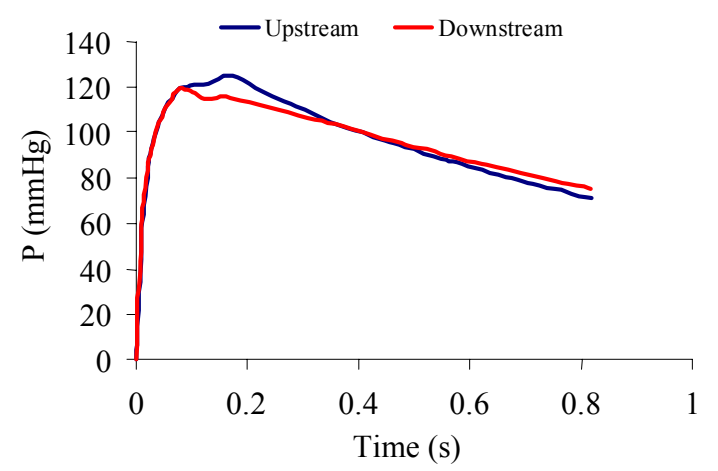

Fig. 14: Calculated pressure for abnormal (obstruction) of right common carotid

Right common carotid pressure graphs for normal and abnormal conditions are shown in Fig. 14.

Also pressure difference between Upstream and Downstream of Obstruction of right common carotid is visible in Fig. 15.

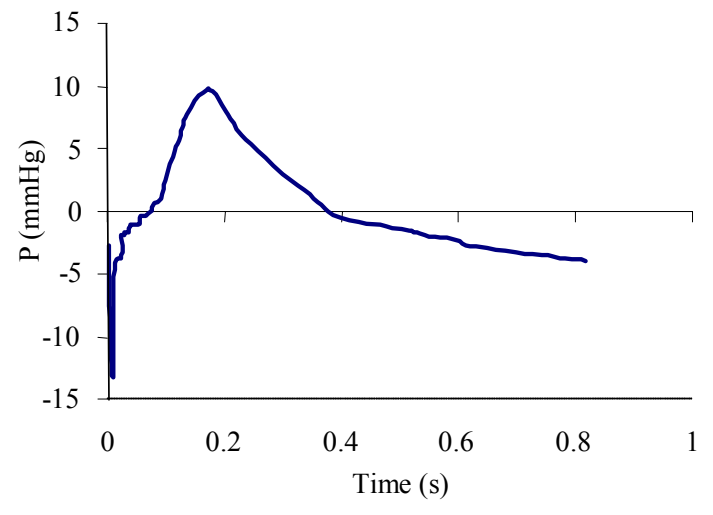

Fig. 15: Pressure Difference between Downstream and Upstream of Right Common Carotid Because of Obstruction

\section{CONCLUSION}

As it was shown in results confirmation the citation of our circuit is quite acceptable.

So it should be noted that using this complex electronic circuit to model human cardiovascular system with its all details, is so useful for studying of blood, different vessels and heart behaviors respect to each other and in different conditions such as health, diseases and abnormities. These abnormities may be obstructions, heart problems, vessels diseases or lots of different other things which out of scope of this study.

Finally it should be said that our circuit has this tendency to be more accurate and useful by adding more compartments and details to it.

\section{REFERENCES}

1. Wang, J.J. and KH. Parker, 2004. Wave propagation in a model of the arterial circulation. J. Biomech., 37: 457-470. DOI:10.1016/j.jbiomech.2003.09.007

2. Tsung-Chieh Lee, Ke-Feng Huang, Ming-Liang Hsiao, Shih-Tsang Tang and Sheunn-Tsong Young, 2004. Electrical lumped model for arterial vessel beds. J. Comput. Methods Programs Biomed., 73: 209-219. DOI:10.1016/S01692607(03)00067-1

3. Mette, S. Olufsen and Ali Nadim, 2004. Deriving lumped models for blood flow and pressure in the systemic arteries. J. Math. Biosci. Eng., 1: 61-88. http://www4.ncsu.edu/ msolufse/OlufsenNadim.pd $\mathrm{f}$

4. Nardinochini, P, G. Pontrelli and L. Teresi, 2005. A one-dimensional model for blood flow in pre stressed vessel. Eur. J. Mechanics, 24: 23-33. DOI:10.1016/j.euromechsol.2004.10.002 
5. Torii, R, M. Oshima and T. Kobayashi, 2005. Computer modeling of cardiovascular fluidstructure interactions with the deformingspatialdomain-time formulation. Comput. Methods Applied Mechanics Eng., 13: 549-57. DOI:10.1016/j.cma.2005.05.050.

6. Wolters, B.J.B.M., M. Emmer, M.C.M. Rutten, G.W.H. Schurink and F.N. Van De Vosse, 2007. Assessment of endoleak significance after endovascular repair of abdominal aortic aneurysms: A lumped parameter model. J. Med. Eng. $\quad$ Phys., 29: 1106-1118. DOI:10.1016/j.medengphy.2006.11.008

7. Mona Abdolrazaghi, Mehdi Navid Bakhsh and Kamran Hassani, 2008. Study of left ventricle pressure-volume loops in abdominal aortic aneruysm utilizing cardiovascular electronic modeling. In: IASTED International Conference Biomedical Engineering, Feb. 13-15, Austria, Innsbruck, $\quad$ pp: 161-166. http://www.actapress.com/Abstract.aspx?paperId= 32670

8. Rideout, V.C., 1991. Mathematical and Computer Modeling of Physiological Systems. 2nd Edn., Prentice Hall, New York, pp: 261. ISBN:0-13563354-0

9. Katia Lagana, Rossella Balossino, Francesco Migiliavacca, Giancarlo Pennati, Edward L. Bove, Marc R. de Leval, Gabriele Dubini, 2005. Multiscale modeling of the cardio vascular system: application to the study of pulmonary and coconary perfusions in the univentricular circulation. Journal of Biomechanics, 38: 1129-1141. DOI:10.1016/j.jbiomech.2004.05.027.
10. Safar, M.E., 2007. Arterial stiffness: A simplified overview in vascular medicine. Adv. Cardiol., 44: 1-18. DOI: 10.1159/000096699

11. Krishnan, B., Chandran, Ajit, P. Yoganathan, Stanley and E. Rittgers, 2007. Biofluid Mechanics Boca Raton. 1st Edn., Taylor and Francis Group, New York. London.

12. Leonard, R. Johnson, 2003. Essential Medical Physiology. 1st Edn., Elsevier Academic Press, pp: 1008. ISBN 0123875846.

13. Lee Waite, 2006. Biofluid Mechanics in Cardiovascular Systems. 1st Edn., McGraw-Hills, New York, pp: 201. ISBN-10: 0071447881

14. Zamir, M., 2005. The Physics of Coronary Blood Flow. Springer, London, $1^{\text {st }}$ Edition.

15. Oguz, Baskurt, K., R. Max Hardeman, W. Michael, Rampling, J. Herbert and Meiselman, 2007. Handbook of Hemorheology and Hemodynamics. IOS Press, pp:455. ISBN 1586037714.

16. Valerie, C., Scanlon and Tina Sanders, 2007. Essentials of Anatomy and Physiology. F.A. Davis Company, New York, $1^{\text {st }}$ Edition. 